\title{
Flexural Waves on Fiber and Fiber Bragg Gratings for WDM Switching and Gain Equalization of Erbium-doped Fiber Amplifiers
}

\author{
Chih-Chung (C. C.) Yang ${ }^{1,2,3}$, Chih-Nan Lin ${ }^{4}$, Chung-Yih Tang', Yan-Ju Chiang 5 , \\ Yean-Woei Kiang ${ }^{1,4}$,Chih-Cheng Chou ${ }^{6}$, Nai-Hsiang Sun ${ }^{6}$, Chih-An Wei ${ }^{6}$, Wen-Fung Liu ${ }^{7}$, I-Ming Liu $^{7}$, \\ Lung-Wei Chung ${ }^{7}$, and Ding-Wei Huang ${ }^{8}$ \\ 'Department of Electrical Engineering National Taiwan University, 1, Roosevelt Road, Sec. 4, Taipei, \\ Taiwan (phone) 886-2-23657624 (fax) 886-2-23652637 (E-mail) ccy@,cc.ee.ntu.edu.tw \\ ${ }^{2}$ Graduate Institute of Electro-Optical Engineering, National Taiwan University, Taipei, Taiwan \\ ${ }^{3}$ Graduate Institute of Electronics Engineering, National Taiwan University, Taipei, Taiwan \\ ${ }^{4}$ Graduate Institute of Communication Engineering, National Taiwan University, Taipei, Taiwan \\ ${ }^{5}$ Department of Electrical Engineering, National Tsing Hua University, Hsinchu, Taiwan \\ ${ }^{6}$ Department of Electrical Engineering, I-Shou University, Kaohsiung, Taiwan \\ ${ }^{7}$ Department of Electrical Engineering, Chung Cheng Institute of Technology, Taoyuan, Tahsi, Taiwan \\ ${ }^{8}$ OES Laboratories, ITRI, Hsinchu, Taiwan
}

\begin{abstract}
Flexural waves on fiber and fiber Bragg gratings generated with applied ultrasounds and loading were used for switching the reflection wavelength of fiber Bragg grating and for controlling the transmission spectra of fiber. By producing lateral vibration of a fiber Bragg grating, which has been etched in cladding, it was found that the reflection wavelength could be switched from the Bragg wavelength to other wavelengths, which corresponded to the conditions of strong coupling between core mode and cladding modes. By adjusting the applied voltage for acoustic waves, the relative strength of different reflection wavelengths could be well adjusted. Theoretical analysis of phase-matching condition indicated that the diameter of etched cladding played a key role in controlling the switched reflection wavelength. Flexural waves were also generated on fiber by applying loading with one or two periodically corrugated plates. By adjusting the orientation of the periodical corrugation, loading level, and the relative position of the two corrugated plates, depressions of transmission spectrum at various wavelengths could be well controlled. The spectral depressions were caused by the coupling between core mode and various cladding modes. Such depressions were used for gain equalization of erbium-doped fiber amplifiers. The equalization operation was more efficient with curved fiber under loading. Control of fiber curvature for various forms of spectral depression will be discussed.
\end{abstract}

Keywords: fiber, fiber Bragg grating, flexural wave, switching, gain equalization

\section{INTRODUCTION}

Application of flexural waves to fiber can produce the effects of long-period grating, which can couple optical signals from the fiber core mode into cladding modes. Such coupling can produce the effects of spectral filtering. The function of spectral filtering can be used for many applications, including amplifier gain equalization [1], add/drop filtering, etc. Spectral filtering can be implemented with long-period fiber gratings of various forms. Effective long-period fiber gratings can be realized with either permanent modification of fiber, such as etching [2] and UV-induced refractive index change [3], or temporary alteration of fiber propagation characteristics. The method of temporary alteration has the advantage of dynamically controlling the function of spectral filtering. Dynamic long-period fiber gratings can be implemented through the application of either an acoustic wave [4]-[7], or periodical loading onto fiber [8]-[10]. In either technique, two possible mechanisms may result in the aforementioned phase matching process, including geometric deformation (micro-bending) and elastooptical effect for periodical refractive index variation of the fiber. However, it is still unclear which one of the two mechanisms dominates the phase matching process.

Acoustics-induced fiber vibration has been used for controlling optical characteristics of bare fiber, fiber couplers and fiber Bragg gratings. Acoustic waves were employed to excite the longitudinal vibration of a fiber Bragg grating for generating side bands of the reflection window [11]. The spectral location and intensity of the side bands were controlled by the acoustic frequency and intensity, respectively. Recently, we have acoustically generated lateral vibration of a fiber Bragg grating to control its reflectivity level after it was written [12]. In this paper, we report the switching of reflection window of 
a fiber Bragg grating through the application of an acoustic wave to the fiber grating. The acoustic wave induced lateral vibration and hence micro bending of the fiber grating. The micro bending served as a long-period grating for coupling the core modes and cladding modes. With this coupling mechanism, the reflection window of the fiber grating can be switched between the Bragg wavelength and the cladding-mode coupling wavelengths. Also, in this paper we report the spectral filtering results of effective long-period gratings implemented by double-sided loading with two identical blocks of periodical grooves. It was found that the positions and depths of transmission spectral depressions varied with the relative phase of the two periodically grooved structures. When the two periodical structures are exactly in phase, i.e., crests are exactly matched, all spectral depressions disappear. This observation leads to the conclusion that geometric deformation is the dominating mechanism for phase matching between the core and cladding modes.

\section{ACOUSTICALLY INDUCED FLEXURAL WAVES FOR GRATING REFLECTION SWITCHING}

Figure 1 shows the setup of the acoustics-modulated device. A fiber Bragg grating was spliced to a 3-dB fiber coupler for measurements. Between the grating section and the coupler, the fiber was laterally glued to the tip of a solid metal (aluminum) horn, whose base was adhered to a piezo-transducer (PZT). The PZT was driven by a voltage source for generating an acoustic wave. The acoustic wave translated onto the fiber and produced transverse vibration. The vibration propagated to the grating section to form micro-bending. To enhance micro-bending effects and control the cladding mode characteristics, the grating section of fiber was etched with HF solution to reduce the cladding diameter from $125 \mu \mathrm{m}$ down to $30 \mu \mathrm{m}$. The grating was $1.7 \mathrm{~cm}$ in length with a slant angle of 2 degrees. The Bragg wavelength was $1541.5 \mathrm{~nm}$ with the Bragg reflectivity about $63 \%$. The uniform part of the etched section had a length of $3.5 \mathrm{~cm}$ with the center coincident with the grating center.

Next, we turned on the continuous-wave voltage source with the frequency at $1.3 \mathrm{MHz}$ and measured the reflection spectra. Parts (a) through (d) of Fig. 2 show the variation of the spectrum at $0,1.8,10$ and $15 \mathrm{~V}$ (root-mean-square values), respectively. We can see that the reflectivity of the $\lambda_{b}$ signal drops from $63 \%$ down to not quite $20 \%$ as the voltage increases up to $15 \mathrm{~V}$. Meanwhile, that of the $\lambda_{\mathrm{s}}$ signal increases from the noise level up to almost $60 \%$. Besides, when the applied voltage is higher than $10 \mathrm{~V}$, the reflectivity of the second cladding-mode emerges. One can observe that switching occurs at around $10 \mathrm{~V}$ of applied voltage with the crossing reflectivity around $47 \%$. The maximum switching speed is at the order of tens $\mathrm{kHz}$. It depends on the acoustic speed and the distance between the acoustic source and the grating.

\section{PHASE MATCHING CONDITIONS FOR ACOUSTICALLY INDUCED MODE COUPLING}

In this section, we intend to provide a detailed explanation for the mode coupling mechanisms in Section 2, particularly the phase-matching conditions, based on the calculations of effective refractive indices of various modes. From the identification of cladding modes, which satisfy the phase-matching conditions for aforementioned couplings, we can calibrate the microbending period. In formulating the problem, we consider the tapered step-index fiber as a cylindrical dielectric waveguide with the core, cladding, and air clothing refractive indices at 1.466, 1.46, and 1, respectively. Because the concerned wavelength range is quite small, material dispersion is neglected in our calculations. The core and cladding radii are $r_{I}=4$ $\mu \mathrm{m}$ and $r_{2}=15 \mu \mathrm{m}$, respectively. The calculations for the effective refractive indices of various modes can be conducted following the standard procedures of solving the dispersion relation. The second column of Table 1 shows the calculated effective refractive indices of various modes at $\lambda_{B}=1541.5 \mathrm{~nm}$. Among them, $\mathrm{LP}_{01}$ mode represents the core mode and all others correspond to cladding modes, as labeled in the first column.

In examining the phase-matching conditions for mode couplings, we first consider the contra-directional coupling, phase-matched by the Bragg grating. Any two modes of contra-directional propagation, with effective refractive indices $n_{a}$ and $n_{b}$, should satisfy the following equation for efficient coupling:

$$
n_{a}+n_{b}=\frac{\lambda}{\Lambda_{B}},
$$

where $\Lambda_{B}$ is the Bragg grating period. In Figs. 3, we plot the dispersive effective refractive indices (defined as $n_{b}$ values) of

various modes as solid lines. Also, $\frac{\lambda}{\Lambda_{B}}-n_{a}$ is plotted as the dashed line. By considering $n_{a}$ as the effective refractive index of the core mode. i.e., $n_{a}=n_{01}$, the intercepted points of the dashed line and solid lines provide the wavelengths of coupling from the forward-propagating core mode into backward-propagating cladding modes. In this calculation, $\Lambda_{B}$ was obtained by considering the Bragg condition, $\lambda_{B}=2 n_{0 I} \Lambda_{B}$, with $\lambda_{B}=1541.5 \mathrm{~nm}$. We obtain $\Lambda_{B}=526.9 \mathrm{~nm}$, which is quite consistent with the period of the mask used for writing this fiber Bragg grating, i.e., about $1.054 \mu \mathrm{m}$. The third column of Table 1 shows these coupling wavelengths obtained from Figs. 3. The discussions here also result in the identification of $\mathrm{LP}_{11}$ for the coupling phenomenon at $\lambda_{C l}=1539.7 \mathrm{~nm}$. For $\lambda_{C 2}=1537.8 \mathrm{~nm}$, the coupling between $\mathrm{LP}_{01}$ and $\mathrm{LP}_{31}$ or $\mathrm{LP}_{41}$ modes is possible. 
To further calibrate the co-directional coupling between the core mode and cladding modes, we first write the phasematching condition as

$$
n_{a}-n_{b}=\frac{\lambda}{\Lambda_{L}}
$$

where $\Lambda_{L}$ is the period of micro-bending caused by the applied flexural wave. In Fig. 4, we plot three lines as $n_{0 l}, n_{l l}$ (solid lines), and $n_{l l}+\lambda / \Lambda_{L}$ (dashed line). Actually, the solid line of $n_{0 l}$ overlaps well with the dashed line of $n_{l l}+\lambda / \Lambda_{L}$ in the concerned wavelength range. In this plotting, $\Lambda_{L}$ was chosen to let the values of $n_{0 l}$ and $n_{l l}+\lambda / \Lambda_{L}$ coincide exactly at $\lambda_{B}=$ $1541.5 \mathrm{~nm}$ because the corresponding coupling is supposed to be the channel of losing energy and reflection reduction at this wavelength. The overlap of the two lines implies that such co-directional coupling can occur within a large wavelength range, including $\lambda_{C I}=1539.7 \mathrm{~nm}$. At this wavelength, the co-directional coupling is supposed to be the channel for the backward $\mathrm{LP}_{11}$ signal power coupled into the backward $\mathrm{LP}_{01}$ mode such that the rising peak was observed in Fig. 1. From this argument, we can obtain $\Lambda_{L}=452 \mu \mathrm{m}$.

Next, we explain the appearance of reflectivity at $\lambda_{C 2}=1537.8 \mathrm{~nm}$ in Fig. 2. The coupling mechanism for this wavelength was identified related to $\mathrm{LP}_{31}, \mathrm{LP}_{41}$, or both modes. For signal coupling into the backward $\mathrm{LP}_{01}$ mode such that the signal power can be detected, a long-period grating with its period shown in the third column of Table 2 is required for satisfying the condition of Eq. (2). As shown in this table, a period of 240 or $194 \mu \mathrm{m}$ is required if $\mathrm{LP}_{31}$ or $\mathrm{LP}_{41}$ mode, respectively, is involved in the coupling process. It is interesting to note that the period of $240 \mu \mathrm{m}$ is close to one-half of the calibrated $\Lambda_{L}=452 \mu \mathrm{m}$. Hence, it is speculated that the co-directional coupling between $\mathrm{LP}_{01}$ and $\mathrm{LP}_{31}$ is due to the secondorder diffraction of the micro-bending. Because of the second-order diffraction nature and the imperfect phase matching of this coupling, the coupling is relatively weaker, compared with the case of $\lambda_{C I}=1539.7 \mathrm{~nm}$. With this result, the period of the micro bending, i.e., $\Lambda_{L}=452 \mu \mathrm{m}$, can be assured. Because in the experiment, the used voltage frequency is $1.3 \mathrm{MHz}$, the flexural wave speed along the $30-\mu \mathrm{m}$ thick fiber is $587.6 \mathrm{~m} / \mathrm{sec}$.

\section{SPECTRAL FILTERING WITH LOADING ONTO FIBER}

Figure 5 shows the experimental setup for double-sided loading. For each groove period, two identical copper blocks of $3 \times 3$ $\times 0.5 \mathrm{~cm}(\mathrm{~L} \mathrm{x} \mathrm{W} \mathrm{x} \mathrm{T)} \mathrm{with} \mathrm{periodical} \mathrm{corrugations} \mathrm{on} \mathrm{the} \mathrm{L} \times \mathrm{W}$ plane were prepared. Square grooves of $400 \mu \mathrm{m}$ in depth and of $50 \%$ in duty cycle were fabricated with mechanical process. Commonly used communication fiber (Corning, SMF-28) was placed between the two corrugated copper blocks. Loading was applied by moving one of the blocks toward the other with a translation stage. The relative position, i.e., relative phase, of the two face-to-face corrugated blocks was controlled by a translation stage moving along another direction. The fiber was aligned along the normal to the groove lines.

The wavelength and depth of the minimums of spectral depressions were recorded when the relative phase of the two corrugated blocks weas varied. Figures 6 and 7 show the variations of depression wavelength and depth with the relative phase when the loading level is about $0.15 \mathrm{~mm}$ stage displacement and the corrugation period is $700 \mu \mathrm{m}$. Here, the relative phase is represented with the stage displacement in mm. In either Fig. 6 or 7, periodical variations with period $700 \mu \mathrm{m}$ can be clearly seen, confirming the 700- $\mu \mathrm{m}$ corrugation period. The other abscissa in either Fig. 6 or 7 indicates the corresponding relative phase. One can clearly see that near the phases of integers of $2 \pi$, all the induced spectral depressions disappear.

Fig. 6 shows the wavelength variations of depression locations. It is noted that the depression wavelengths reach the longest values when the relative phase is an odd integer of $\pi$. In this situation, the signal coupling is expected to be strongest because the depression depth reaches the maximum, as shown in Fig. 7. The tuning range in varying the relative phase is about $10 \mathrm{~nm}$. However, as shown in Fig. 7, the spectral depression depth drops fast when the relative phase approaches an integer of $2 \pi$. The insignificant loading effect in the case that the relative phase is an integer of $2 \pi$ has an important implication. Application of a flexural wave to a fiber, either with an acoustic wave or loading, may produce two mechanisms for inducing mode coupling: geometric deformation and pressure-induced elasto-optical effect. The former forms micro bending for phase matching. The later generates periodical refractive index variation for phase matching. From the results described above, we can conclude that geometric deformation is the dominating mechanism for phase matching in mode coupling in our loading configuration.

\section{CONCLUSIONS}

In this paper, we have reported the implementation the coupling between the core and cladding modes near a fiber Bragg grating by acoustically generating micro bending of the fiber. The micro-bending phase matching worked together with the grating phase matching to result in the reflectivity decrease at the Bragg wavelength and increase at cladding-mode coupling wavelengths. Also, we have calculated the effective refractive indices of the core mode and various cladding modes of a single-mode, step-index fiber, with its cladding tapered down to several tens of $\mu \mathrm{m}$ in radius. The calculation results were used to identify the phase-matching conditions for contra-directional and co-directional couplings between the core mode and 
cladding modes. In addition, we have demonstrated the spectral filtering effects of fiber by double-sided loading with identical periodical corrugations. It was found that the filtering wavelength and strength were dependent on the relative phase of the two periodical corrugations. Particularly, when the relative phase is zero (crest-to-crest), spectral filtering effects disappear completely. Such results lead to the conclusion that geometric deformation, instead of pressure-induced elastooptical effect, is the dominating mechanism for generating spectral filtering functions in the double-sided loading configuration.

\section{ACKNOWLEDGEMENTS}

This research was supported by National Science Council, The Republic of China, under the grants of NSC 89-2218-E-002094, NSC 89-2218-E-002-095, and NSC 89-2215-E-002-051.

\section{REFERENCES}

1. M. Tachibana, R. I. Laming, P. R. Morkel, and D. N. Payne, IEEE Photo. Tech. Lett. 3, 118 (1991).

2. S. A. Vasiliev, E. M. Dianov, D. Varelas, H. G. Limberger, and R. P. Salathe, Opt. Lett. 21, 1830 (1996).

3. A. M. Vengsarkar, P. J. Lemaire, J. B. Judkins, V. Bhatia, T. Erdogan, and J. E. Sipe, J. Lightwave Tech. 14, 58 (1996).

4. R. Faced, C. Alegria, M. N. Zervas, and R. I. Laming, IEEE J. Select. Topic in Quantum Electron. 5, 1278 (1999).

5. D. W. Huang, W. F. Liu, C. W, Wu and C. C. Yang, IEEE Photo. Tech. Lett. 12, 176 (2000).

6. W. F. Liu, I. M. Liu, L. W. Chung, D. W. Huang, and C. C. Yang, Opt. Lett. 25, 1319 (2000).

7. A. Diez, T. A. Birks, W. H. Reeves, B. J. Mangan, and P. St. J. Russell, Opt. Lett. 25, 1499 (2000).

8. R. C. Youngquist, J. L. Brooks, and H. J. Shaw, Opt. Lett. 8, 656 (1983).

9. R. C. Youngquist, J. L. Brooks, and H. J. Shaw, Opt. Lett. 9, 177 (1984).

10. S. Savin, M. J. F. Digonnet, G. S. Kino, and H. J. Shaw, Opt. Lett. 25, 710 (2000).

11. W. F. Liu, P. St. J. Russell and L. Dong, J. Lightwave Tech., 16, 2006 (1998).

12. D. W. Huang, W. F. Liu, C. W, Wu and C. C. Yang, IEEE Photon. Tech. Lett., 12, 176 (2000).

Table 1 Matching of the spectral dips in Fig. 2 with the calculated coupling (contra-directional coupling caused by the Bragg grating) wavelengths of various cladding modes.

\begin{tabular}{|c|c|c|c|}
\hline Mode labels & $\begin{array}{c}\text { Effective refractive indices of various } \\
\text { modes at } \lambda_{B}=1541.5 \mathrm{~nm}\end{array}$ & Calculated coupling wavelengths & $\begin{array}{c}\text { Wavelengths of major dips } \\
\text { in experiments }\end{array}$ \\
\hline $\mathrm{LP}_{01}$ & 1.46278 & $1541.5 \mathrm{~nm}$ & $1541.5 \mathrm{~nm}$ \\
\hline $\mathrm{LP}_{11}$ & 1.45938 & $1539.7 \mathrm{~nm}$ & $1539.7 \mathrm{~nm}$ \\
\hline $\mathrm{LP}_{21}$ & 1.45771 & $1538.8 \mathrm{~nm}$ & $1538.8 \mathrm{~nm}$ \\
\hline $\mathrm{LP}_{31}$ & 1.45635 & $1538.3 \mathrm{~nm}$ & $1537.8 \sim 1538 \mathrm{~nm}$ \\
\hline $\mathrm{LP}_{41}$ & 1.45483 & $1537.3 \mathrm{~nm}$ & $1537.3 \mathrm{~nm}$ \\
\hline $\mathrm{LP}_{51}$ & 1.45308 & $1536.4 \mathrm{~nm}$ & $1536.4 \mathrm{~nm}$ \\
\hline $\mathrm{LP}_{61}$ & 1.45112 & $1535.4 \mathrm{~nm}$ & $1535.3 \mathrm{~nm}$ \\
\hline $\mathrm{LP}_{71}$ & 1.44893 & $1534.3 \mathrm{~nm}$ & $1534.1 \mathrm{~nm}$ \\
\hline $\mathrm{LP}_{81}$ & 1.44653 & $1533.1 \mathrm{~nm}$ & $1532.7 \mathrm{~nm}$ \\
\hline $\mathrm{LP}_{52}$ & 1.44629 & $1532.9 \mathrm{~nm}$ & \\
\hline
\end{tabular}

Table 2 Required period of long-period grating for co-directional coupling between the core mode and a cladding mode for $\lambda_{C 2}$ wavelength reflection.

\begin{tabular}{|c|c|c|}
\hline Mode labels & Effective refractive index at $\lambda_{C 2}=1537.8 \mathrm{~nm}$ & Required period \\
\hline $\mathrm{LP}_{01}$ & 1.46279 & $\sim$ \\
\hline $\mathrm{LP}_{31}$ & 1.45637 & $240 \mu \mathrm{m}$ \\
\hline $\mathrm{LP}_{41}$ & 1.45486 & $194 \mu \mathrm{m}$ \\
\hline
\end{tabular}




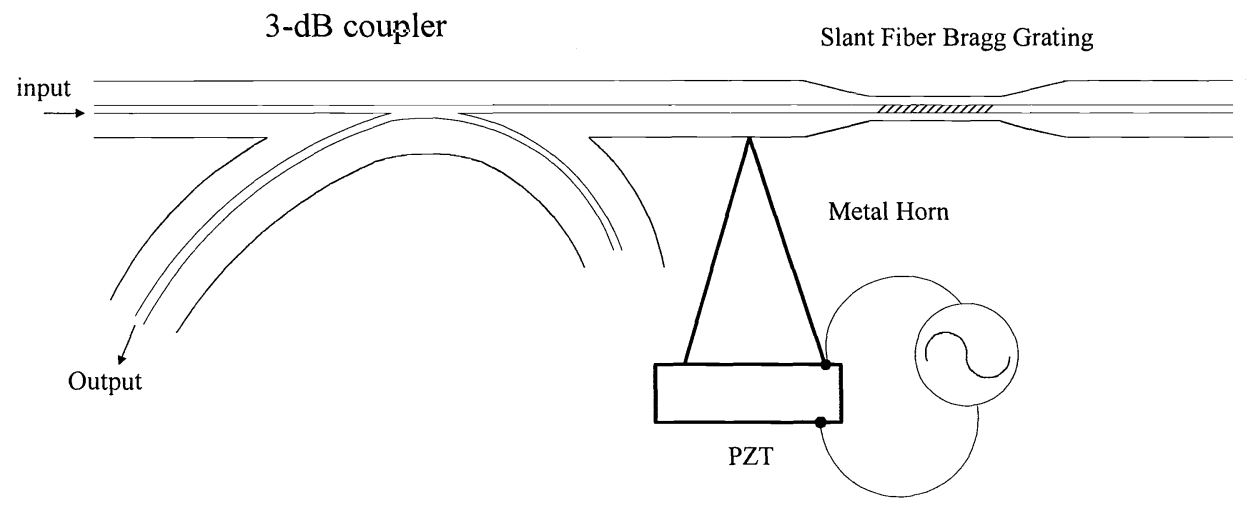

Fig. 1 Setup for acoustically induced grating reflection switching.
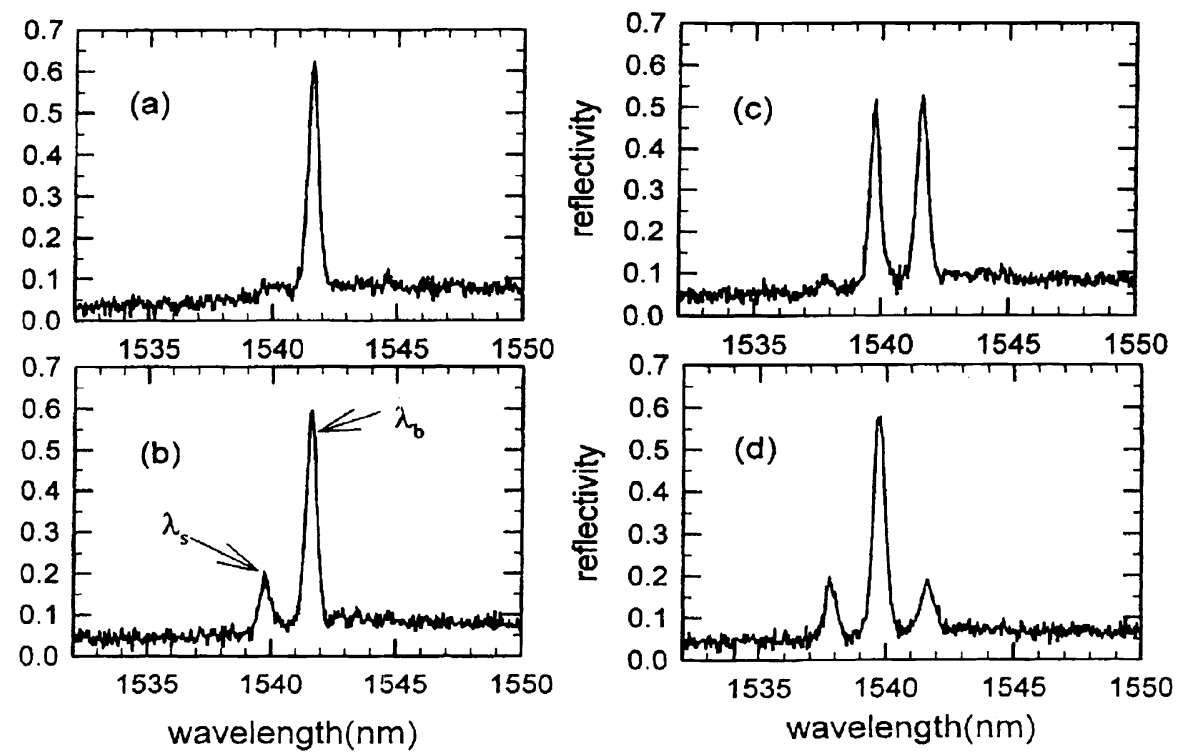

Fig. 2 Grating reflection switching results.

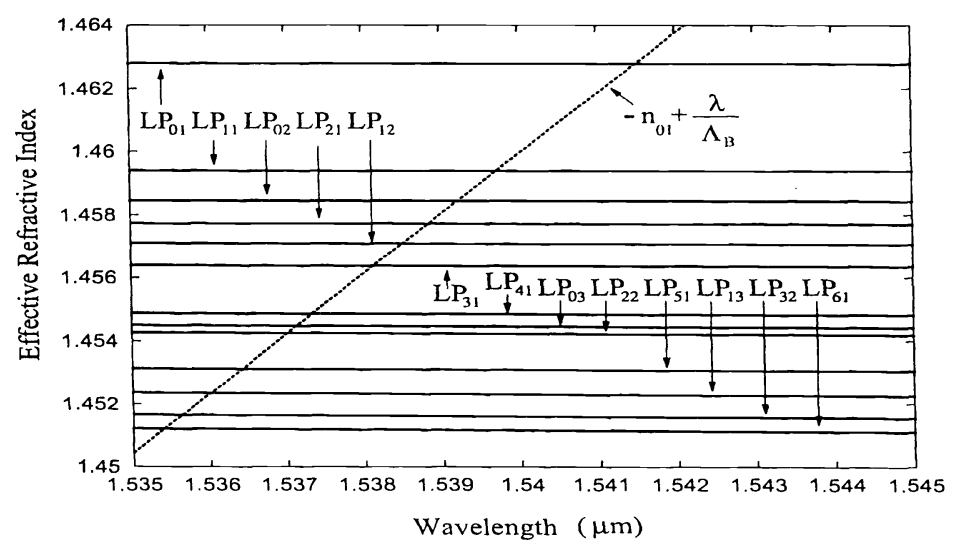

Fig. 3 Wavelength dependencies of the effective refractive indices of various modes (solid lines) and $\lambda / \Lambda_{B}-n_{01}$ (dashed line). The intercepted points indicate the wavelengths of contra-directional coupling between the core mode and various cladding modes. 


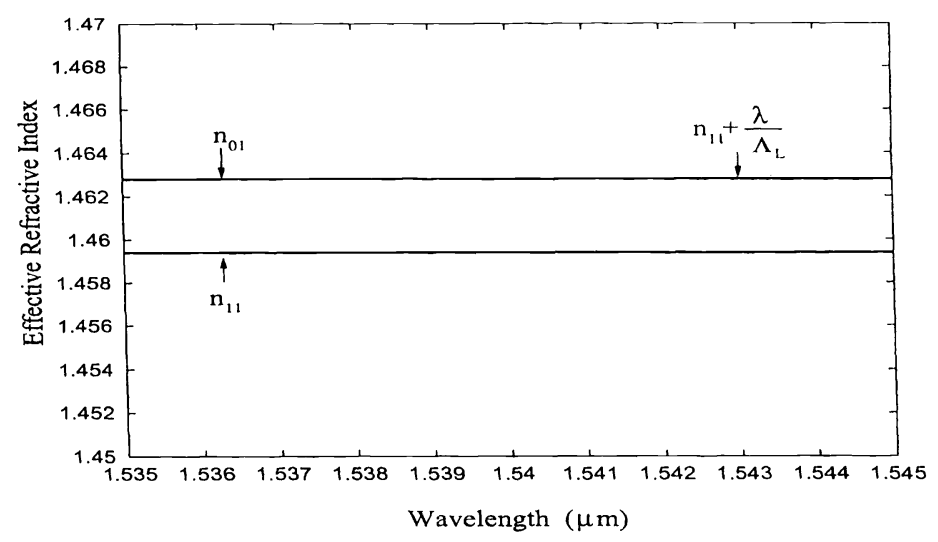

Fig. 4 Wavelength dependencies of the effective refractive indices of the core mode $\left(n_{01}\right)$, the first cladding mode $\left(n_{l l}\right)$, and that of $n_{1 I}+\lambda / \Lambda_{L}$. The lines of $n_{0 l}$ and $n_{I I}+\lambda / \Lambda_{L}$ overlap quite well in the concerned wavelength range.

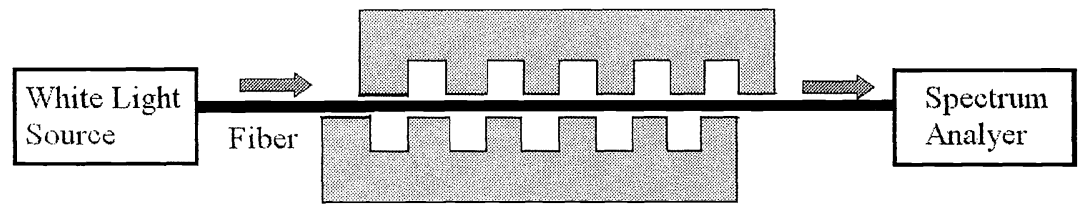

Fig. 5 Experimental setup of double-sided loading onto fiber for spectral filtering function.

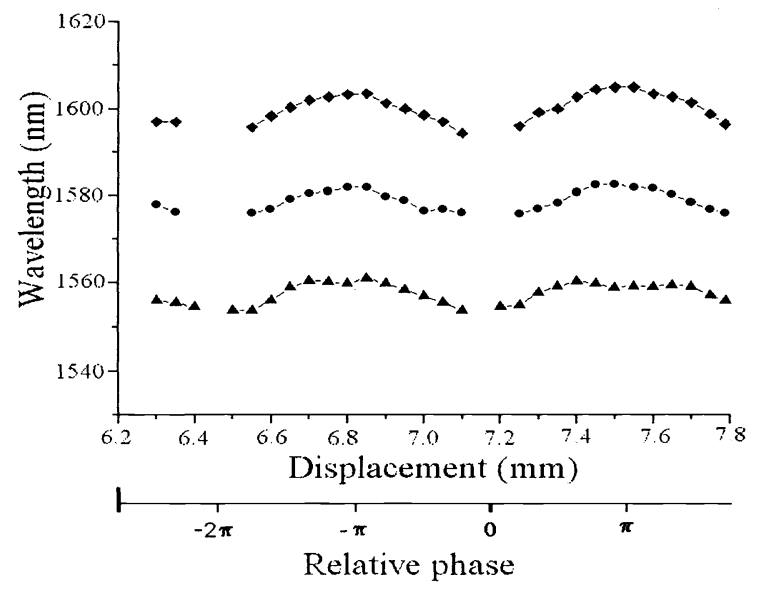

Fig. 6 Variations of the wavelengths of spectral depression minimums with the relative phase of the two periodical corrugations. Two abscissas represent the physical displacement and the relative phase of the two periodical corrugations. 


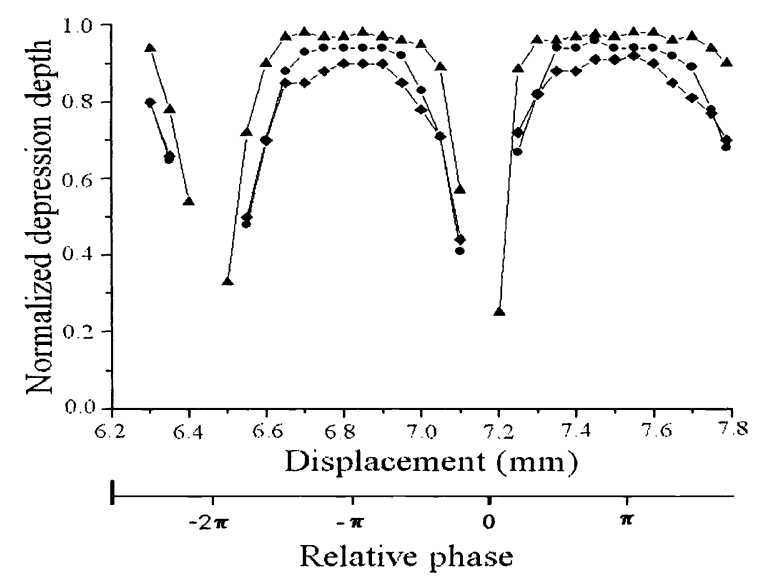

Fig. 7 Variations of the depths of spectral depression minimums with the relative phase of the two periodical corrugations. The three data sets correspond to those of Fig. 6. 